\title{
Capsular Polysaccharides Produced by the Bacterial Pathogen Burkholderia pseudomallei
}

\author{
Shauna L. Reckseidler-Zenteno
}

Additional information is available at the end of the chapter

http://dx.doi.org/10.5772/50116

\section{Introduction}

Polysaccharide capsules are structures found on the cell surface of a broad range of bacterial species. The polysaccharide capsule often constitutes the outermost layer of the cell, and therefore is often involved in mediating direct interactions between the bacteria and its environment. It is due to these interactions that polysaccharide capsules have been implicated as important virulence factors for many bacterial pathogens.

Bacterial extracellular polysaccharides (EPS) may be classified as either capsular polysaccharides (CPS), where the polysaccharide is intimately associated with the cell surface, or as slime polysaccharides, where the polysaccharide is loosely associated with the cell [1]. Differentiation between these forms is difficult since CPS may be released from the cell, giving the appearance of a slime polysaccharide [1]. In turn, distinguishing between CPS and other cell surface polysaccharides, such as O-antigenic moieties of lipopolysaccharide (LPS), may also be difficult, since CPS may be found associated with LPS [1].

Capsular polysaccharides are highly hydrated molecules that are over 95\% water [2]. They are often linked to the cell surface of the bacterium via covalent attachments to either phospholipid or lipid-A molecules, although some CPS may be associated with the cell in the absence of a membrane anchor [1,3]. Capsular polysaccharides can be either homo- or heteropolymers composed of repeating monosaccharides joined by glycosidic linkages [4]. The multiple hydroxyl groups present within each monosaccharide may be involved in the formation of the glycosidic bond, therefore, any two monosaccharides may be joined in a number of configurations, which leads to large structural diversity among CPS types. In the case of human pathogens, a large number of different capsule serotypes have been identified, and certain CPS or K-antigens have been associated with specific infections [4]. For example, the Escherichia coli K1 antigen, a homopolymer of $\alpha 2,8$-linked $\mathrm{N}$ acetylneuraminic acid (NeuNAc), is the major cause of neonatal meningitis [5]. While 
bacterial species may demonstrate great structural diversity in synthesizing capsules, chemically identical capsular polysaccharides may also be synthesized by different bacterial species. The Neisseria meningitidis group B capsular polysaccharide is identical to the K1 polymer of E. coli, and the E. coli K18, K22, and K100 antigens have the same constituents and structure as the Haemophilus influenza serotype b capsule [6, 7]. The conservation of CPS types between bacterial species raises interesting questions regarding the evolution of capsules and the transmission and acquisition of capsule biosynthesis genes [4].

The genetic loci necessary for the production of bacterial capsules are primarily clustered at a single chromosomal locus, which allows for the coordinate regulation of a large number of genes that may be involved in both the biosynthesis and export of capsular polysaccharides $[4,1]$. In most bacterial species, the capsule gene clusters demonstrate conserved genetic organization. The capsules of $E$. coli have been classified into four groups based on genetic organization and biosynthetic criteria and capsules of other bacteria may resemble these prototypes. Group 1 capsules include the E. coli K30 capsule and the capsules of Klebsiella sp. and Erwinia sp. [8]. Group 2 capsules include the E. coli $\mathrm{K} 1$ and $\mathrm{K} 5$ capsules, as well as the capsules produced by Neisseria sp. and Haemophilus sp. Group 3 capsules include the E. coli K10 capsule. Group 4 capsules include the capsules of E. coli K40 and 0111. Both group 2 and group 3 capsule gene clusters are organized into 3 regions. Regions 1 and 3 are involved in the export and modification of the capsular polysaccharides and are conserved between members of the group, while region 2 contains the genes responsible for the biosynthesis of the capsule and is usually serotype specific $[4,8]$. Generally these regions are organized into one transcriptional unit and the regions within the capsule locus are divergently transcribed $[4,8]$. In addition, some genes within a region may be translationally coupled, such as the $k p s U$ and $k p s C$ genes in group 2 E. coli capsules, and the kpsM and kpsT genes in group 3 E. coli capsules, which allows for balanced expression of two proteins [4].

The $\mathrm{A}+\mathrm{T}$ composition of capsule gene clusters is often significantly higher than the rest of the chromosome, suggesting a common ancestry of capsule genes in gram-negative bacteria [9]. It is likely that these $A+T$ rich regions have been horizontally transferred between bacterial species. In addition, the A+T ratio of region 2 DNA of group 2 E. coli capsule gene clusters compared to regions 1 and 3 confirms that capsule diversity has been achieved in part through the acquisition of different region 2 sequences [4].

The production of a polysaccharide capsule is widespread in pathogenic bacteria. A number of functions have been assigned to bacterial capsules including: prevention of dessication, adherence, resistance to non-specific host immunity, resistance to specific host immunity, and mediating the diffusion of molecules through to the cell surface $[4,1]$.

Capsules may form a hydrated gel around the surface of the bacterial cell, which may protect the bacteria from the harmful effects of dessication [10]. This may increase the survival of encapsulated bacteria outside of the host, promoting the transmission of pathogenic bacteria from one host to another [4]. Mucoid isolates of E. coli, Acinetobacter calcoaceticus, and Erwinia stewartii are more resistant to drying than isogenic nonmucoid strains [11]. Studies with E. coli have shown that the expression of genes encoding for the 
colanic acid capsule is increased by dessication [11]. In addition, alginate production by Pseudomonas aeruginosa is triggered by high osmolarity, which may be a consequence of dessication [12].

Capsular polysaccharides may promote adherence of bacteria to both surfaces and other bacterial cells, which may facilitate colonization of a particular niche and may lead to the formation of biofilms [13]. Cell-surface polysaccharides have been shown to mediate the attachment of bacterial cells to one another, leading to biofilm formation and persistence of the organisms during colonization $[1,14]$.

Capsular polysaccharides are one of the components responsible for resistance to the nonspecific immunity of the host. The presence of a capsule is thought to confer resistance to non-specific host defense mechanisms such as complement and complement-mediated opsonophagocytosis [4]. Bacterial capsules may resist complement-mediated killing by providing a permeability barrier to complement components, which masks the underlying cell surface structures that activate complement [15]. The capsule may also act in concert with O-antigens to confer resistance to complement-mediated killing [16]. As a result, a combination of cell surface structures is responsible for conferring resistance to killing by the complement cascade [4]. Finally, capsules are responsible for resistance to complementmediated opsonophagocytosis. This resistance may be due to steric effects, which results in the capsule acting as a barrier between the $\mathrm{C} 3 \mathrm{~b}$ deposited on the bacterial surface and the $\mathrm{C} 3 \mathrm{~b}$ receptors present on phagocytes [4]. Alternatively, the resistance to opsonophagocytosis may be due to the net negative charge of the polysaccharide capsule [17].

Capsules may also confer resistance to the specific immune response of the host. Although most capsular polysaccharides can elicit an immune response, some capsules are poorly immunogenic [4]. Examples of such capsules include those containing NeuNAc, such as the E. coli $\mathrm{K} 1$ capsule or the capsule of Neisseria meningitidis serogroup B, and the E. coli K5 capsule, which is similar to desulfoheparin $[18,19]$. Because these capsules are structurally similar to polysaccharides encountered on host tissue, these capsules are poorly immunogenic, and elicit a poor antibody response in the host [20].

Burkholderia pseudomallei, the causative agent of melioidosis, is a gram-negative, facultatively anaerobic, motile bacillus that is commonly found in the soil and stagnant waters in a number of regions around the world, particularly in areas that fall between $20^{\circ}$ north and $20^{\circ}$ south of the equator [21, 22]. Infection by B. pseudomallei is often due to either direct inoculation into wounds and skin abrasions or to inhalation of contaminated material [22, 23, 24,]. This would explain the prevalence of the disease among rice farmers as well as helicopter pilots in the Vietnam War who developed melioidosis due to inhalation of contaminated dust [24]. Melioidosis may present as an acute pneumonia or an acute septicemia, which is the most severe form of the disease. The disease may also manifest as a chronic infection involving long-lasting suppurative abscesses in numerous sites in the body. Infection with B. pseudomallei may even result in a subclinical infection and remain undetected for a number of years. Both the chronic and subclinical forms generally remain undiagnosed until activated by a traumatic event or a decrease in immunocompetence [25]. B. pseudomallei is 
inherently resistant to a number of antibiotics, and even with aggressive antibiotic therapy, the mortality rate remains high, and the incidence of relapse is common [26, 27].

At the time our studies were initiated some cell-associated antigens had been identified and characterized in B. pseudomallei. Cell-associated antigens include exopolysaccharide (EPS) and lipopolysaccharide (LPS) $[28,29,30]$. The EPS produced by B. pseudomallei was determined to be an unbranched polymer of repeating tetrasaccharide units with the structure -3)-2-O-acetyl- $\beta$-D-Gal $p-(1-4)-\alpha$-D-Gal $p-(1-3)-\beta$-D-Gal $p-(1-5)-\beta-D-K D O p-(2-[31,32]$. The role of EPS in virulence was not known, but sera from patients with melioidosis had been shown to contain antibodies against EPS [30]. Two other EPS structures were also identified; a branched 1,4-linked glucan polymer ((CP-1a) and a triple-branched heptasaccharide repeating unit composed of rhamnose, mannose, galactose, glucose, and glucuronic acid (CP-2) [33]. The genes involved in the synthesis of these capsules, and the role of these capsules in virulence had not been identified. The LPS of B. pseudomallei was structurally characterized and reported to contain two types of O-polysaccharide moieties termed type I O-PS and type II O-PS [34, 35]. Type II O-PS was found to be an unbranched heteropolymer with repeating D-glucose and L-talose residues with the structure -3$)-\beta-\mathrm{D}$ glucopyranose-(1-3)-6-deoxy- $\alpha$-L-talopyranose-(1-. Type II O-PS had been shown to be involved in serum resistance [36]. Type II O-PS mutants also demonstrated reduced virulence in three animal models of B. pseudomallei infection [36]. Type I O-PS was determined to be an unbranched homopolymer with the structure -3)-2-O-acetyl-6-deoxy- $\beta$ D-manno-heptopyranose-(1-, however, the role for this polysaccharide in infection had not been defined, nor the genes responsible for its biosynthesis been identified.

B. thailandensis is a nonpathogenic soil organism originally isolated in Thailand [37]. Based on biochemical, immunological, and genetic data, B. pseudomallei and B. thailandensis are closely related species. However, these two organisms differ in a number of ways and have been classified into two different species [38]. The rRNA sequence of $B$. thailandensis differs from that of $B$. pseudomallei by 15 nucleotides, and there are significant differences in genomic macrorestriction patterns between these organisms [39]. The biochemical profiles of these two species differ in that $B$. thailandensis can utilize L-arabinose whereas B. pseudomallei does not $[38,40]$. The most distinct difference between these two species, however, is their relative virulence. The $50 \%$ lethal dose (LD50) for B. pseudomallei in the Syrian hamster model of acute melioidosis is $<10$ organisms, whereas the $\mathrm{LD}_{50}$ for $B$. thailandensis is approximately $10^{6}$ organisms [38]. It has also been shown that the two species can be differentiated based on their propensity to cause disease in humans. Environmental strains isolated in Thailand that are able to assimilate L-arabinose are not associated with human infection, whereas clinical isolates are not able to utilize L-arabinose [41].

\section{Subtractive hybridization to identify Capsular Polysaccharide 1 (CPS I)}

To identify the genetic determinants that confer enhanced virulence in B. pseudomallei, a method combining subtractive hybridization, insertional mutagenesis, and animal virulence studies was developed [42]. Subtractive hybridization was carried out between the virulent $B$. pseudomallei and the weakly virulent $B$. thailandensis in order to isolate DNA sequences 
encoding for virulence determinants unique to $B$. pseudomallei. The genomic DNA sample from B. pseudomallei containing the sequences of interest was known as the tester DNA, and genomic DNA from $B$. thailandensis, the reference sample, was called the driver DNA. Tester and driver DNAs were digested and subjected to two rounds of hybridization. The remaining unhybridized sequences were considered tester-specific sequences. To enrich for tester-specific sequences, excess driver DNA was added in the hybridizations. The testerspecific sequences were then amplified by PCR and cloned into the plasmids PPCR or pZErO-2.1. Screening of the subtraction library revealed a number of DNA sequences unique to $B$. pseudomallei. Fifteen distinct plasmid inserts from the library were sequenced. The DNA inserts ranged from 100 to $800 \mathrm{bp}$ in length and were found to contain an average $\mathrm{G}+\mathrm{C}$ content of approximately 44 to $52 \%$, which is considerably lower than the $68 \% \mathrm{G}+\mathrm{C}$ content of the $B$. pseudomallei chromosome. The DNA sequences were analyzed using the NCBI BLASTX program. One of the plasmid inserts, pDD1015, was found to share limited homology with $\mathrm{WbpX}$, a glycosyltransferase, from Pseudomonas aeruginosa [43].

\subsection{Demonstration that CPS $\mathrm{I}$ is important for the virulence of $B$. pseudomallei}

The 373-bp DNA insert from pDD1015 was cloned into a mobilizable suicide vector, pSKM11 [44]. The resulting plasmid, pSR1015, was mobilized into wildtype B. pseudomallei $1026 \mathrm{~b}$ to create the mutant strain SR1015. Since the insert from pDD1015 was found to demonstrate homology to a glycosyltransferase from $P$. aeruginosa, it was postulated that it might encode a protein involved in carbohydrate synthesis. Since three carbohydrate structures had been previously purified and characterized, antibodies to each of these polysaccharides were available. To define the phenotype of SR1015, an ELISA was performed with the EPS-specific monoclonal antibody 3015, and B. pseudomallei 1026b and SR1015 were both found to contain EPS [45]. SR1015 was also shown to contain type II O-PS and to be serum resistant. Immunogold electron microscopy studies using rabbit polyclonal sera specific for a type I O-PS-flagellin conjugate was performed on the parent strain, 1026b, and SR1015 (Figure 1). B. pseudomallei 1026b reacted with antibodies to both flagellin and type I O-PS, as was evident by the distribution of gold particles around the bacterial surface and extending out along the flagella (Figure 1A). Unlike B. pseudomallei 1026b, SR1015 reacted only with the antibodies to flagellin, as the gold particles were found associated only with the flagella (Figure 1B). B. thailandensis, the negative control, did not react with the antibodies either to flagellin or to type I O-PS (Figure 1C). B. stabilis LMG7000 was also shown to react to the antibodies to type I O-PS, indicating this organism may produce a similar capsule (Figure 1D). Western blot analysis of proteinase K-digested whole cells from $B$. pseudomallei 1026b, B. thailandensis E264, and B. pseudomallei SR1015 using rabbit polyclonal sera raised to O-PS-flagellin protein conjugate confirmed the lack of type I O-PS in SR1015. Type I and type II O-PS were stained in B. pseudomallei 1026b, while only type II O-PS was stained in the lanes corresponding to B. pseudomallei SR1015 and B. thailandensis. These results indicated that we had identified and insertionally inactivated a gene involved in the synthesis of the type I O-PS of B. pseudomallei [42]. SR1015 was tested for virulence in the Syrian hamster model of acute septicemic melioidosis. The LD50 for SR1015 after $48 \mathrm{~h}$ was $3.5 \times 10^{5} \mathrm{CFU}$, while the $\mathrm{LD}_{50}$ of the parent strain, $1026 \mathrm{~b}$, was $<10$ CFU. The LD50 for SR1015 was similar to that for 
the weakly virulent $B$. thailandensis $\left(6.8 \times 10^{5} \mathrm{CFU}\right)$ [42]. This demonstrated that SR1015 is severely attenuated for virulence in this animal model of melioidosis and that type I O-PS is a major virulence determinant of B. pseudomallei. We later determined that the type I O-PS was a capsular polysaccharide (CPS I), not an O-PS moiety, which will be discussed below.
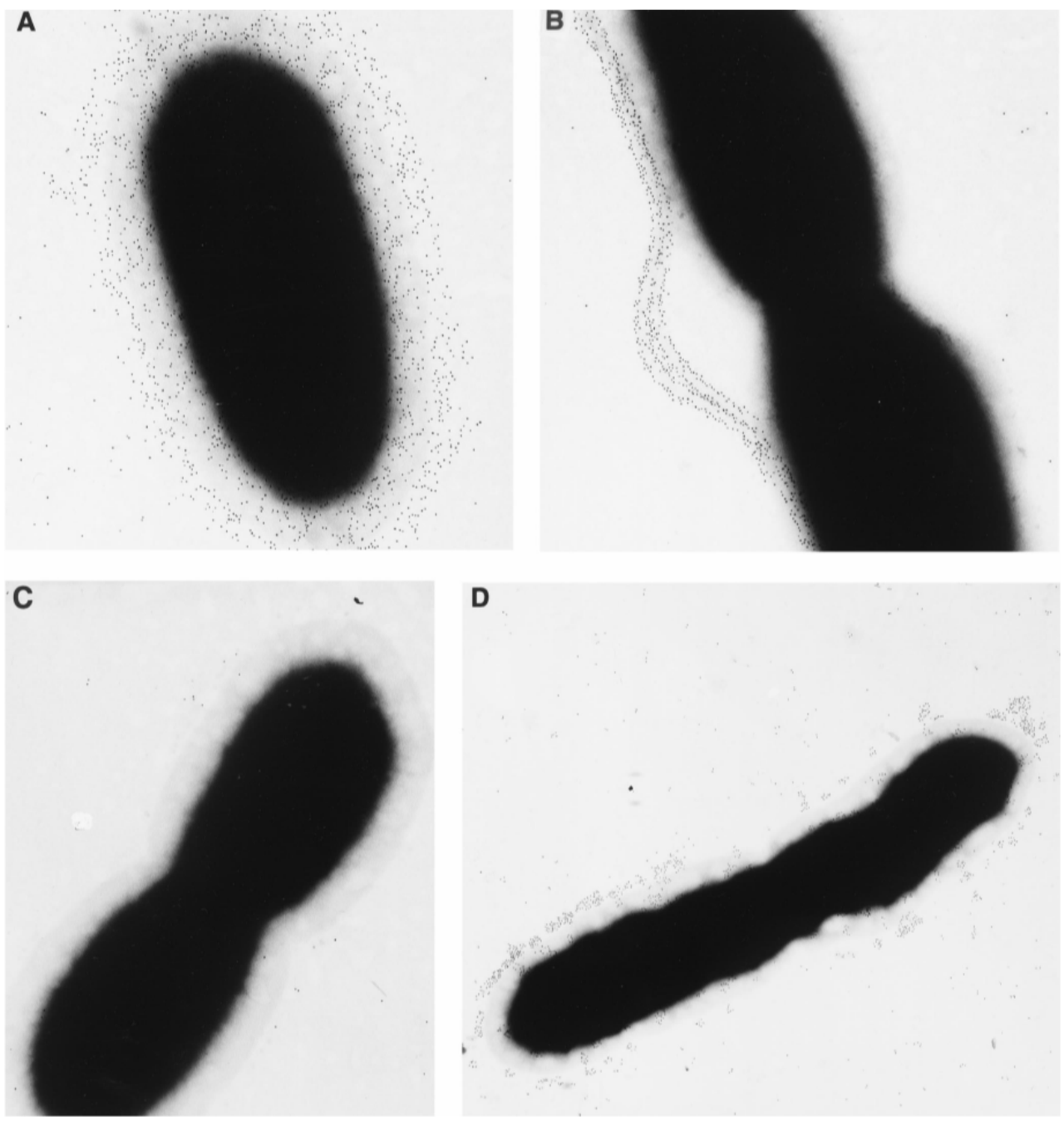

Figure 1. Immunogold electron microscopy of B. pseudomallei 1026b (A) and SR1015 (B), B. thailandensis E264 (C), and B. stabilis LMG7000 (D). Bacteria were reacted with polyclonal rabbit antiserum directed against an O-PS-flagellin protein conjugate absorbed with B. thailandensis E264 to remove the antibodies directed against type II O-PS, washed, and reacted with a goat anti-rabbit IgG-gold (5 nm) conjugate. Original magnification x330,000. 


\subsection{Cloning and sequencing of the genetic loci required for CPS I production and export}

Two methods were used to clone the genes involved in the production and export of type I O-PS. The DNA flanking the insertion of pSR1015 was cloned from SR1015 and sequenced. We also used transposon mutagenesis to clone the genes involved in production of the polysaccharide; this was done to obtain any unlinked genes that may be involved in polysaccharide production. Approximately 1,300 transposon mutants were screened for loss of type I O-PS by ELISA. Six mutants were identified, and the DNA flanking the transposon insertion was cloned and sequenced. The Tn5-OT182 mutants SLR5, SLR8, SLR13, SLR18, and SLR19 mapped to the same region of the chromosome. Sequence analysis of the cloned fragments revealed the presence of 26 potential open reading frames involved in the synthesis and export of type I O-PS [42]. The open reading frames that predicted proteins involved in polysaccharide biosynthesis were found to demonstrate homology to proteins involved in the synthesis of a polysaccharide structure composed primarily of mannose. The other reading frames in the locus predicted proteins involved in the transport of capsular polysaccharides in a variety of bacteria, particularly those that produce group 2 and group 3 capsular polysaccharides [8]. The genes responsible for the production of type I O-PS were found to be similar to other loci encoding for capsular polysaccharides in that they are divergently transcribed [4]. The gene cluster involved in the production of this polysaccharide is also similar to group 3 capsule gene clusters in that there are no genes encoding $\mathrm{KpsF}$ and $\mathrm{KpsU}$, which are present in group 2 capsule gene clusters [8]. However, the organization of the B. pseudomallei type I O-PS gene cluster differs in that it does not contain two export regions flanking a single biosynthetic region as seen in other group 3 capsule polysaccharide clusters [46]. The biosynthetic genes identified are not organized into one continuous transcriptional unit; instead, $w c b B, m a n C$, and $w c b P$ are separated from the rest of the biosynthetic genes. The overall $\mathrm{G}+\mathrm{C}$ content of this region is about $58 \%$, lower than the $\mathrm{G}+\mathrm{C}$ content of the rest of the chromosome $(68 \%)$. The low $\mathrm{G}+\mathrm{C}$ content in these clusters suggests that polysaccharide genes have a common origin and may have been transferred horizontally between species [9]. The genes involved in the production of this polysaccharide were named according to the bacterial polysaccharide gene nomenclature scheme [47]. The gene products associated with this cluster are shown in Figure 4. Mutations constructed in a number of these genes confirmed their role in the production of this polysaccharide [42].

\subsection{CPS I was originally identified as a Type I O-polysaccharride moiety}

The polysaccharide with the structure -3)-2-O-acetyl-6-deoxy- $\beta$-D-manno-heptopyranose-(1was originally isolated and characterized as an O-PS component of LPS in B. pseudomallei and was designated type I O-PS [35]. However, our results suggested that this polysaccharide was a capsule rather than an O-PS moiety. The genes involved in the production of this capsule demonstrated strong homology to the genes involved in the production of capsular polysaccharides in many organisms, including N. meningitidis, $H$. influenzae, and E. coli. In addition, the export genes associated with this cluster are not associated with the previously characterized O-PS gene cluster [36]. Western blot analysis of 
proteinase $\mathrm{K}$ cell extracts and silver staining demonstrated that this polysaccharide has a high molecular mass $(200 \mathrm{kDa})$ and lacks the banding pattern seen with O-PS moieties. Studies by our laboratory indicated that mutants in the production of the core oligosaccharide of the LPS are still capable of producing this polysaccharide [48]. Based on the above criteria and the genetic similarity to group 3 capsules, we proposed that this polysaccharide is a group 3 capsule and designated this capsule CPS I. This conclusion was further supported by Isshiki et al who separated this polysaccharide from a smooth lipopolysaccharide preparation of B. pseudomallei [49].

\section{Role of the B. pseudomallei capsule in virulence}

The role of CPS I in the pathogenicity of B. pseudomallei was investigated by performing further animal studies, serum bactericidal assays, complement protein $\mathrm{C} 3 \mathrm{~b}$ deposition assays, and radio-labelled phagocytic assays [50]. These experiments were facilitated by constructing a deletion strain harbouring a mutation in one of the CPS I genes and by complementation of this strain. An in-frame deletion was constructed in $w c b B$, a gene which encodes a glycosyltransferase, resulting in the capsule-minus strain SZ210. To confirm the role of $w c b B$ in the biosynthesis of capsule, SZ210 was complemented by the introduction of a wild-type copy of the $w c b B$ gene cloned into the mobilizable broad-host-range plasmid pBHR1 (MoBiTec). Western blot analysis of proteinase K-digested whole cells was performed using mouse monoclonal antibody directed to B. pseudomallei capsule to assess capsule production by these strains. Similar to the capsule minus strain SR1015 and B. thailandensis E264, which is known to lack this capsule, SZ210 was found to be negative for CPS I production, as indicated by the absence of a $200 \mathrm{kDa}$ band that is present for wild-type 1026b. Complementation of SZ210 by providing the wild type $w c b B$ gene in trans restored capsule production. Whole-cell extracts from the complemented strain SZ210(pSZ219) reacted to the capsule antibody producing the $200-\mathrm{kDa}$ band corresponding to the $B$. pseudomallei capsule.

\subsection{Production of CPS I correlates with clinical infection}

To establish a correlation between CPS I production and clinical infection a number of strains of $B$. pseudomallei isolated from a variety of clinical specimens were tested for capsule production by western blot analysis with polyclonal rabbit antisera to B. pseudomallei CPS I. Out of the 55 clinical strains tested for capsule production, 52 were found to produce this capsule. Three strains, 420a, 415c, and 375a were found to be negative for capsule production, similar to $B$. thailandensis E264. However, one of the capsule genes, wzt2, was successfully amplified from these three strains and following inoculation in the animal model, all three of these strains were found to produce capsule by western blot analysis. This indicated that CPS I production may be regulated in some strains and its expression may be induced in vivo. Therefore all of the 55 clinical strains of B. pseudomallei tested were found to produce capsule, establishing a 100\% correlation between CPS I production and clinical infection [51]. 


\subsection{CPS I promotes survival of B. pseudomallei in vivo}

Syrian golden hamsters were inoculated intraperitoneally with $10^{1}$ to $10^{5}$ cells of either wild type B. pseudomallei 1026b, capsule mutants SR1015 and SZ210, or the complemented strain SZ210(pSZ219). One group of animals inoculated with SR1015 also received $100 \mu \mathrm{g}$ of purified B. pseudomallei capsule. After $48 \mathrm{~h}$, the $\mathrm{LD}_{50}$ values were calculated, and the blood of the infected animals was diluted and plated for bacterial quantitation. The addition of purified capsule significantly increased the virulence of the capsule mutant strain SR1015. The $\mathrm{LD}_{50}$ value was calculated to be $34 \mathrm{CFU}$, similar to the $\mathrm{LD}_{50}$ value of wild-type $B$. pseudomallei 1026b ( $<10 \mathrm{CFU})$. In contrast, the LD50 value for SR1015 without the addition of purified capsule was calculated to be $3.5 \times 10^{5} \mathrm{CFU}, 10,000$ - fold higher than when capsule was added to the inoculum. In addition, purified capsule enhanced the survival of SR1015 in the blood. Bacteria could not be detected in the blood of hamsters inoculated with SR1015 alone. However, the number of SR1015 CFU recovered from the blood of infected animals was $9.0 \times 10^{2} \mathrm{CFU} / \mathrm{ml}$ when capsule was added to the inoculum, an almost-1,000-fold increase. This number was comparable to the number of wild-type B. pseudomallei 1026b bacteria recovered from the blood. The addition of capsule was not toxic to the hamsters, as hamsters inoculated with $100 \mu \mathrm{g}$ of purified capsule alone survived for the duration of the experiment without any ill effects. The LD50 value for the capsule mutant strain SZ210 containing an in frame deletion of the $w c b B$ gene was calculated to be $9.6 \times 10^{4} \mathrm{CFU}$, and the number of bacteria in the blood was determined to be $10 \mathrm{CFU} / \mathrm{ml}$. Complementation of this strain restored virulence in the animal model, resulting in an LD50 value of $12 \mathrm{CFU}$, comparable to that of wild type B. pseudomallei 1026b. Furthermore, the number of bacteria in the blood of animals infected with the complemented strain, SZ210(pSZ219), was determined to be $4.9 \times 10^{5} \mathrm{CFU} / \mathrm{ml}$, similar to the number of bacteria recovered from animals infected with $1026 \mathrm{~b}$ [50].

To further demonstrate the role of the capsule in infection by B. pseudomallei, an experiment was designed to investigate differences in tissue distribution between the capsule mutant strain and the wild type in infected hamsters. Animals were inoculated with $10^{2} \mathrm{CFU}$ of either wild-type B. pseudomallei $1026 \mathrm{~b}$ or the capsule mutant SR1015. At different time points, the animals were sacrificed, and the numbers of bacteria in the blood, liver, lungs, and spleen of each animal were determined. As seen in Figure 2, the numbers of $B$. pseudomallei 1026b and SR1015 bacteria were nearly undetectable at $12 \mathrm{~h}$ (Figure 2A). By 24 $\mathrm{h}$, the numbers of $1026 \mathrm{~b}$ bacteria recovered from the blood, lung, liver, and spleen increased, while SR1015 was detected only in the spleen (Figure 2B). By 48 h, very high numbers of $1026 \mathrm{~b}$ bacteria were recovered from all of the organs taken, representing a dramatic increase compared to the inoculum (Figure 2C). In contrast, all of the organs taken from hamsters infected with SR1015 contained fewer bacteria (Figure 2C). Of particular interest was the fact that the number of SR1015 bacteria recovered from the blood at $48 \mathrm{~h}$ was lower than in the inoculum, suggesting that the capsule mutant was cleared from the blood more effectively than the wild type. The number of SR1015 bacteria recovered from the spleen was higher than the number of SR1015 bacteria in the blood, suggesting that SR1015 was being cleared from the blood and sequestered in the spleen. The difference in virulence between the two 
strains can be attributed to capsule production, since the CPS I mutant strain was found to have a growth rate similar to that of the wild-type strain 1026b [50].

A.

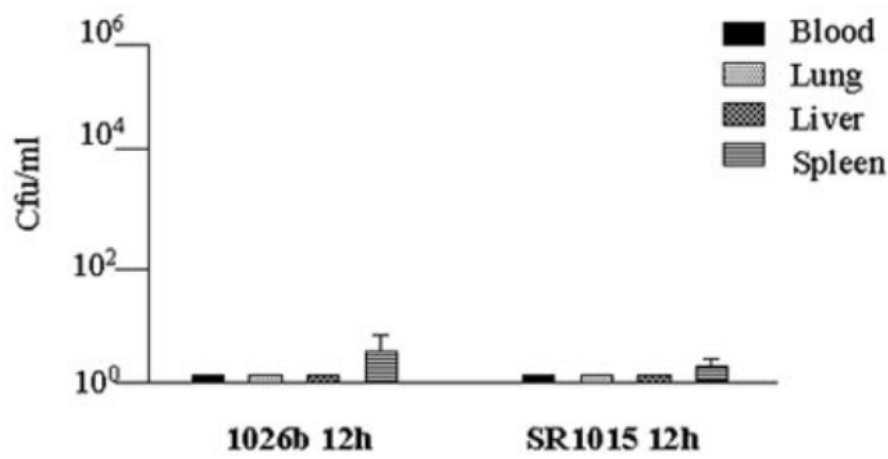

B.

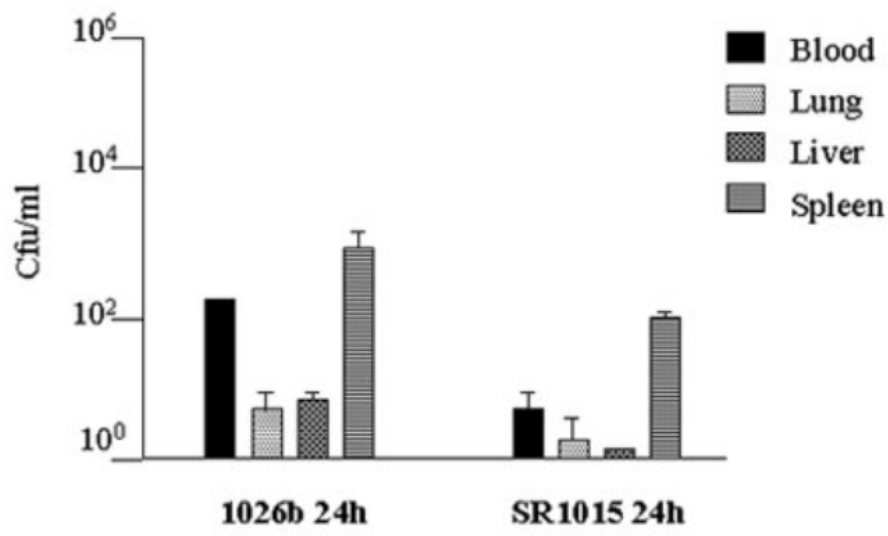

C.

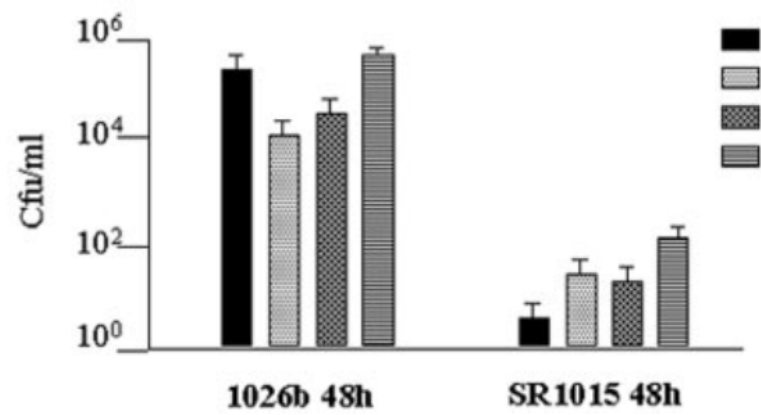

Figure 2. Differences in tissue distribution between B. pseudomallei strains $1026 \mathrm{~b}$ and SR1015 in the Syrian hamster model of acute melioidosis. Female Syrian hamsters (three per group) were inoculated intraperitoneally with $10^{2} \mathrm{CFU}$ of either strain, and at 12,24 , and $48 \mathrm{~h}$, two groups of animals were sacrificed and bacterial quantitation of the tissues was determined. The data represent the average number of bacteria found in each tissue and the standard deviation for a given time point. 


\subsection{CPS I production by B. pseudomallei is responsible for persistence in the blood by inhibiting complement}

To define the role of the capsule for persistence in the blood, serum bactericidal assays were performed with the addition of purified capsule to determine if capsule had an effect on the survival of serum-sensitive strains of B. pseudomallei. For these experiments, we utilized a double mutant that we constructed in the laboratory, SLR5, which lacks both capsule and Opolysaccharide, since the capsule mutant SR1015 was previously found to be serum resistant [42]. The survival of SLR5 was extremely poor when incubated in the presence of $30 \%$ normal human serum (NHS). However, the addition of purified capsule increased the survival of SLR5 in NHS. The addition of $50 \mu \mathrm{g}$ of capsule to the reaction increased the numbers of SLR5 to $5.9 \times 10^{1} \mathrm{CFU} / \mathrm{ml}$, and the addition of $100 \mu \mathrm{g}$ of capsule increased the survival of SLR5 by nearly 1,000-fold to $1.9 \times 10^{3} \mathrm{CFU} / \mathrm{ml}$. Furthermore, pre-incubation of $30 \% \mathrm{NHS}$ with $100 \mu \mathrm{g}$ of capsule (PI-CPS) before the addition of bacteria increased the survival of SLR5 100,000-fold to $4.4 \times 10^{6} \mathrm{CFU} / \mathrm{ml}$. This was similar to the survival of SLR5 when incubated with serum that was heat-inactivated (HI-NHS). These effects were found to be specific to capsule, since the addition of 50 or $100 \mu \mathrm{g}$ of purified B. pseudomallei O-PS or preincubation of the serum with OPS did not increase the survival of serum-sensitive SLR5 [50].

Since capsule mutants of B. pseudomallei are serum resistant in that they are not susceptible to lysis by the membrane attack complex (MAC) because they still produce O-PS, we postulated that the ability of the capsule to enhance survival in the blood could be due to its ability to inhibit $\mathrm{C} 3 \mathrm{~b}$ deposition and opsonization. To investigate the effect of capsule on $\mathrm{C} 3 \mathrm{~b}$ deposition, the amount of $\mathrm{C} 3 \mathrm{~b}$ deposited on the surfaces of wild-type B. pseudomallei $1026 \mathrm{~b}$ and the capsule mutant, SR1015, in the presence of serum was determined by Western blot analysis using a mouse monoclonal antibody specific to human complement factor C $3 \mathrm{~b}$. The deposition of $\mathrm{C} 3 \mathrm{~b}$ was found to be more pronounced in the capsule mutant SR1015 than in the wild type in both 10 and 30\% NHS. Similar results were observed with the capsule mutant SZ210, a strain containing an in-frame deletion of the $w c b B$ gene. More C3b was detected when SZ210 was incubated in both 10 and 30\% NHS than with 1026b. Optical densitometry measurements were performed in order to quantitate the difference in $\mathrm{C} 3 \mathrm{~b}$ deposition between the strains. The average amount of C3b deposited on the surfaces of SR1015 and SZ210 bacteria was 3.5-fold higher than for 1026b in 10\% NHS and 2.5-fold higher in $30 \%$ NHS. In addition, there was a shift in the molecular mass of C3b, which normally runs at $185 \mathrm{kDa}$, indicating a covalent attachment of the molecule to the bacterial surface. The nature of this attachment was not investigated; however, $\mathrm{C} 3 \mathrm{~b}$ is thought to covalently attach to the bacterial surface through an ester or amide linkage [50,52].

Immunofluorescence microscopy analysis was also performed to demonstrate the difference in $\mathrm{C} 3 \mathrm{~b}$ deposition between the capsule mutant and the wild type. The same experiment described above was performed, and samples were reacted with the mouse monoclonal antibody to human complement factor $\mathrm{C} 3 \mathrm{~b}$, except that the samples were reacted with a secondary antibody conjugated to Cy3 and stained with DAPI for visualization of bacterial cells. As shown in Figure 3, the B. pseudomallei capsule mutant SR1015 demonstrated more reactivity to the antibody to human $\mathrm{C} 3 \mathrm{~b}$ in the presence of serum than the wild-type 1026b. 
This is evident from the red fluorescence that corresponds to the C3b bound to the bacterial surface surrounding the blue DAPI-stained cells seen when the capsule mutant was incubated in the presence of $10 \%$ NHS (Figure 3D to F). In contrast, the amount of red fluorescence surrounding the DAPI-stained wild-type cells was minimal in the presence of $10 \%$ NHS (Figure 3A to C). There was a dramatic difference in the amount of C3b deposited on the surface of the capsule mutant compared to the wild type, which was detectable after only 15 min of incubation of the bacteria with human serum (Figure 3B and E). By $60 \mathrm{~min}$, there was some $\mathrm{C} 3 \mathrm{~b}$ deposition on wild-type B. pseudomallei; however, there was still more $\mathrm{C} 3 \mathrm{~b}$ deposited on the surface of the capsule mutant (Figure 3C and F) [50]. This experiment was not performed with $30 \%$ NHS due to excessive clumping of the samples during the fixation process, which resulted in inconsistent and poor staining of the cells. Western blot analysis was also performed to determine the amount of complement factor C3b deposition on the surface of B. thailandensis E264, a related nonpathogenic organism. The amount of $\mathrm{C} 3 \mathrm{~b}$ deposition in B. thailandensis E264 was more pronounced than with B. pseudomallei $1026 \mathrm{~b}$ and was similar to the amount of $\mathrm{C} 3 \mathrm{~b}$ deposited on the surface of the capsule mutant, B. pseudomallei SR1015, in the presence of human serum. The amount of C3b deposition that occurred on the surface of $B$. thailandensis was expected, since the organism is known to lack this capsule $[42,50]$.

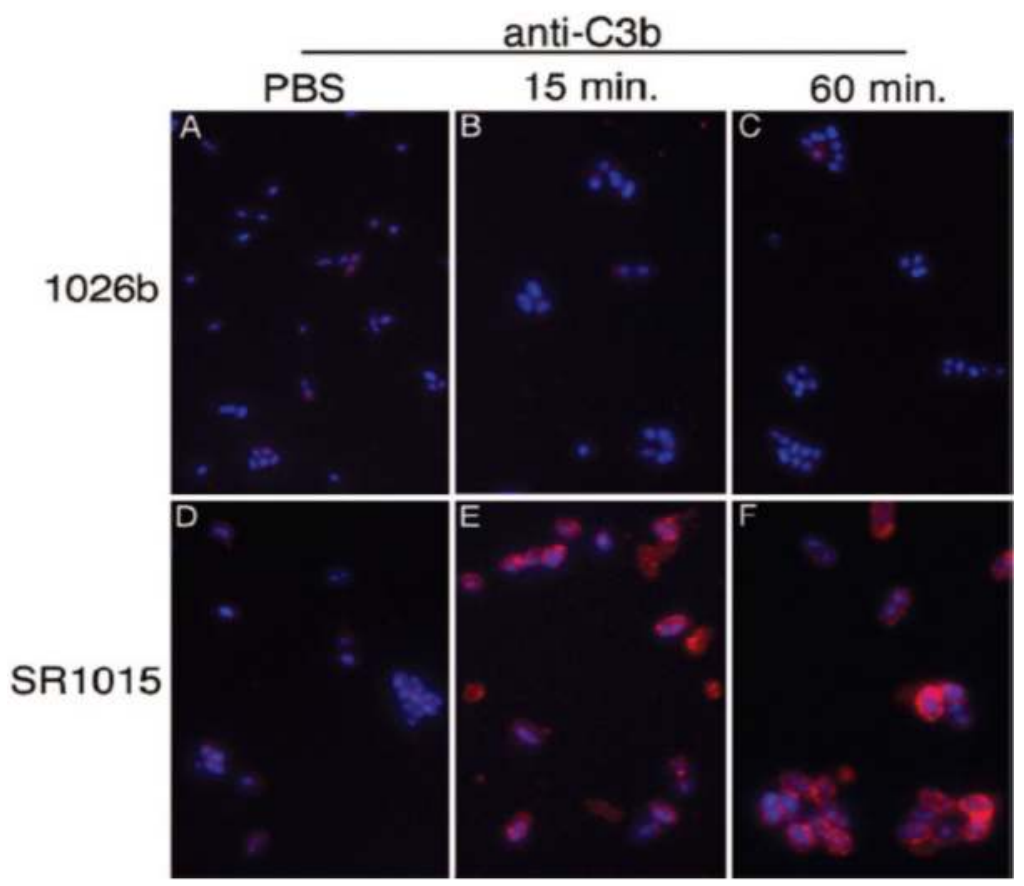

Figure 3. Immunofluorescence microscopy analysis of decreased complement factor $\mathrm{C} 3 \mathrm{~b}$ deposition in $10 \%$ normal human serum by B. pseudomallei capsule. B. pseudomallei $1026 \mathrm{~b}$ and SR 1015 were incubated in $10 \%$ normal human serum (NHS), reacted with a mouse monoclonal antibody to human complement factor $\mathrm{C} 3 \mathrm{~b}$, reacted with a rabbit anti-mouse IgG conjugated to Cy3 (Jackson Laboratories), and stained 
with DAPI for visualization of whole bacterial cells (Sigma). (A) B. pseudomallei 1026b incubated in PBS; (B) 1026b incubated in 10\% NHS for $15 \mathrm{~min}$; (C) 1026b incubated in 10\% NHS for $60 \mathrm{~min}$; (D) $B$. pseudomallei SR1015 incubated in PBS; (E) SR1015 incubated in 10\% NHS for 15 min; (F) SR1015 incubated in $10 \%$ NHS for $60 \mathrm{~min}$. The blue fluorescence indicates the DAPI stained bacteria, and the red fluorescence indicates the binding of complement factor $\mathrm{C} 3 \mathrm{~b}$ to the bacterial surface.

\subsection{The B. pseudomallei capsule CPS I reduces phagocytosis}

The capsule mutant SR1015 was phagocytosed more significantly by PMNL than the wildtype strain. The proportion of wild-type B. pseudomallei 1026b phagocytosed in the presence of $10 \%$ NHS was $35.9 \%$, while the proportion of the capsule mutant SR1015 phagocytosed was $51.7 \%(P<0.001)$. When each strain was incubated in the presence of $30 \%$ NHS, $59.3 \%$ of the wild-type strain $1026 \mathrm{~b}$ was phagocytosed by the PMNL after 30 min compared to $82.3 \%$ for the capsule mutant $(P<0.001)$ [50].

\subsection{B. pseudomallei CPS I expression is elevated in the presence of $30 \%$ normal human serum}

The lux reporter strain B. pseudomallei SZ211 was constructed by cloning an internal fragment of the $w c b B$ gene into pGSV3-lux, a suicide vector containing the lux operon from Photorhabdus luminescens [53]. Absorbance (OD540) and luminescence (in relative light units) measurements were taken every $2 \mathrm{~h}$. Capsule expression was higher in the presence of M9 plus $1 \%$ glucose plus 30\% normal human serum (NHS) and M9 plus 1\% glucose plus 30\% heat-inactivated serum (HI-NHS) than in M9 plus $1 \%$ glucose alone. The increase in light production of SZ211 in the presence of serum supports the requirement for capsule for survival in serum. The strain B. pseudomallei SZ213 was constructed by cloning an internal region of the wbiA gene, which encodes an $O$-acetyltransferase required for $O$-acetylation of the O-PS component of B. pseudomallei LPS [36]. Light production of this strain was measured under the same conditions to determine whether LPS expression was induced in the presence of serum. Similar to the capsule, LPS expression was elevated in the presence of both $30 \%$ NHS and 30\% HI-NHS. The levels of expression of both capsule and LPS were not significantly different in NHS and HI-NHS, suggesting that the environment of the serum may be required for induction of gene expression rather than complement [50].

\section{Other capsules produced by B.pseudomallei}

Sequence analysis of the completed genome of B. pseudomallei revealed four operons with the predicted function of capsular polysaccharide biosynthesis and export [54]. One of these operons, with the gene identifiers BPSL2786-2810, corresponds to the previously characterized mannoheptose capsule designated CPS I $[42,50]$. Three other operons were identified. These three capsule operons in the genome of $B$. pseudomallei were further analyzed using the BLAST program and Artemis. The operons are illustrated in Figure 4. The operon consisting of the genes BPSS0417-0429, was designated CPS II (Figure 4B). Another operon, BPSS1825-1835, was designated CPS III and the predicted homologues 
were investigated further (Figure 4C). A fourth operon, CPS IV, was found to contain genes that may be involved in the synthesis of a capsule with the gene identifiers BPSL2769-2785 (Figure 4D) [54, 55].

A.

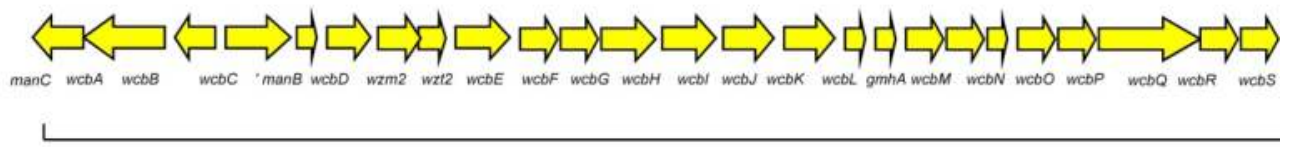

$33 \mathrm{kh}$

B.

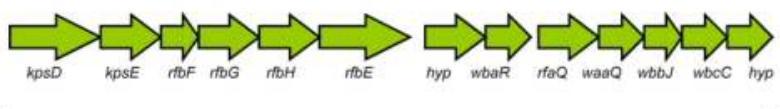

$16 \mathrm{~kb}$

C.

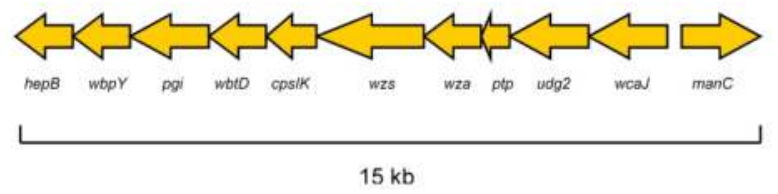

D.

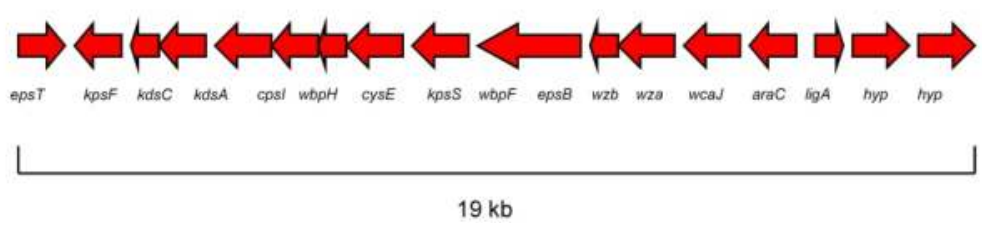

Figure 4. Organization of the chromosomal regions containing the genes comprising the B. pseudomallei capsule operons. The direction of transcription is represented by arrows, and the gene names demonstrating the highest degree of homology to the B. pseudomallei open reading frames are indicated. The relative sizes of each locus are indicated. (A) B. pseudomallei capsule cluster I (CPS I). (B) $B$. pseudomallei capsule cluster II (CPS II). (C) B. pseudomallei capsule cluster III (CPS III). (D) B. pseudomallei capsule cluster IV (CPS IV).

\subsection{Distribution of capsule loci between three Burkholderia species}

Comparative analysis of the genomes of three Burkholderia species, B. pseudomallei, B. mallei and $B$. thailandensis, was performed to determine whether all of the predicted B. pseudomallei capsule operons were present in B. mallei and B. thailandensis as well. CPS II, III, and IV were found to be present in B. pseudomallei and B. thailandensis, but not B. mallei. This is in contrast to CPS I, which is present in B. pseudomallei and B. mallei, but not B. thailandensis $[42,50,54$, $55,56,57]$. CPS II was found to be identical between $B$. pseudomallei and $B$. thailandensis, but $B$. thailandensis was found to contain two flanking hypothetical genes not present in $B$. pseudomallei. The CPS II genes were found to be deleted entirely from B. mallei. A large 
chromosomal region ranging from open reading frames BPSS0404 to BPSS0491 including CPS II was shown to be deleted in B. mallei compared to B. pseudomallei and replaced with a large chromosomal region containing open reading frames BMAA0555 (IS407A orfB) to BMAA1784, a unique hypothetical protein not found in B. pseudomallei. The two genomes align with the presence of the alkyl hydroperoxidase reductase genes $\operatorname{ahpC}$ and $\operatorname{ahpF}$, but these are organized in the opposite orientation in B. mallei compared to B. pseudomallei. The entire CPS III operon and flanking genes were shown to be the same in both B. pseudomallei and $B$. thailandensis. In contrast, the majority of the CPS III cluster was deleted from B. mallei with the exception of the wcaJ and manC genes, as well as the two flanking hypothetical genes on one side, and another hypothetical gene on the other side of the deleted region. The deletion of the CPS III genes in B. mallei was found to be replaced with the IS407A orfA and orfB genes. The entire CPS IV region was found to be replaced in $B$. mallei and flanked by two IS407A elements. The open reading frame BPSL2785, which encodes a hypothetical protein, is present in B. mallei (BMA2284.1) ATCC 23344 as well as a number of other $B$. mallei strains, but is organized in the opposite orientation. The genomes of $B$. pseudomallei, $B$. thailandensis, and B. mallei all diverge upstream of the CPS IV region, but all three organisms were found to align at the location of the omp $A$ and hypothetical genes [55].

\subsection{CPS III does not contribute to the virulence of B. pseudomallei}

In order to assess the role of CPS III in virulence a mutant in the CPS III operon was tested for virulence compared to wild type B. pseudomallei in the Syrian hamster model of melioidosis. Syrian golden hamsters were inoculated intraperitoneally with $10^{1}$ to $10^{3}$ cells of either wild-type B. pseudomallei $1026 \mathrm{~b}$ or the capsule mutant SZ1829. After $48 \mathrm{~h}$, the LD50 values were determined. SZ1829 had LD50 values of $<10 \mathrm{CFU}$, identical to that of wild type $B$. pseudomallei, indicating that this capsule is not required for virulence. In addition, the bacterial load in the blood of the infected hamsters was similar to that of wild-type and significantly higher than that of the non-pathogenic B. thailandensis E264 and the CPS I mutant, B. pseudomallei SR1015, both of which are incapable of establishing bacteremia [37, 50]. This indicates that CPS III does not contribute to persistence in the blood. Similar results were obtained for CPS II and CPS IV mutants, but we went on to further characterize CPS III.

\subsection{Expression of CPS III in host and environmental conditions}

A $l u x$ reporter strain was constructed in the CPS III operon by cloning an internal fragment of one of the genes into pGSV3-lux, a suicide vector containing a promoterless $l u x$ operon from Photorhabdus luminescens [53]. Regulation of this capsule in an environment similar to that encountered in the host was determined by growing the lux reporter strain, SZ1829, in the presence of M9 plus 1\% glucose versus M9 plus 1\% glucose plus 30\% normal human serum (NHS). Absorbance (OD540) and luminescence (in relative light units) measurements were taken every hour. The expression of CPS III (SZ1829) was higher in M9 plus 1\% glucose alone compared to M9 plus 1\% glucose plus 30\% NHS. The expression of SZ1829 was 3-4 fold lower in 30\% NHS. This was in contrast to the expression of CPS I (SZ211) (see section 3.5), which was significantly more highly expressed in 30\% NHS at a level of 3-4 fold 
compared to growth in M9 plus 1\% glucose alone [55]. Although CPS III demonstrated higher expression initially in 30\% NHS, this may have been due to the fact that the addition of NHS caused precipitation in the media which affected the optical density of the cultures.

The expression of the lux operon in reporter strains SZ211 (CPS I-) and SZ1829 (CPS III-) was also measured in water to determine whether CPS III was induced in this environment. Overnight cultures of SZ211 and SZ1829 were inoculated into sterile water and incubated at $37^{\circ} \mathrm{C}$ without shaking. Capsule expression was determined as described above, but the luminescence/absorbance calculations for water were compared to the values for these strains when grown in LB. CPS III was found to be induced in water compared to LB. The expression of SZ1829 was found to be significant with an increase of 2-3 fold over the course of the experiment. The expression of CPS I was found to be greater than 4 fold higher in LB compared to water [55].

Microarray analysis of capsule expression was performed using a low-density DNA microarray. RNA was isolated from the livers and lungs of hamsters infected with $B$. pseudomallei and from B. pseudomallei grown in LB. The results of the microarray experiment are shown in Table 1 [55]. The level of gene expression, or fold change, is represented as the ratio of gene expression in the hamster compared to growth in LB. As shown in Table 1, CPS III genes were not found to be significantly expressed in vivo since most of the fold changes were determined to be less than 2-fold. Many of the genes had negative fold change values, indicating that these genes are suppressed in the host environment. The highest fold change result was 2.337866 for BPSS1827, a predicted glucose-6-phosphate isomerase, which is still much lower than the fold changes observed for CPS I genes, which were significantly higher [58].

\begin{tabular}{|l|l|l|}
\hline Gene ID & Predicted function & $\begin{array}{l}\text { Fold Change } \\
\text { (in vivo vs. } \text { in vitro })\end{array}$ \\
\hline BPSS1825 & Glycosyltransferase & 1.757155 \\
\hline BPSS1826 & Glycosyltransferase & 0.093565 \\
\hline BPSS1827 & Glucose-6-phosphate isomerase & 2.337866 \\
\hline BPSS1828 & Glycosyltransferase & -0.66607 \\
\hline BPSS1829 & Glycosyltransferase & -0.18061 \\
\hline BPSS1830 & Capsule export, tyrosine-protein kinase & -0.30655 \\
\hline BPSS 1831 & Capsule export, outer membrane protein & 0.725849 \\
\hline BPSS1832 & Transport, tyrosine-protein phosphatase & 1.52665 \\
\hline BPSS1833 & UDP-glucose-6-dehydrogenase & -0.71411 \\
\hline BPSS1834 & Sugar transferase & -0.07963 \\
\hline BPSS 1835 & Mannose-1-phosphate guanyltransferase & -0.75075 \\
\hline
\end{tabular}

${ }^{*}$ Note: Gene expression representative of the liver and lungs of infected hamsters.

Table 1. Microarray analysis of B. pseudomallei CPS III expression following intraperitoneal inoculation in the hamster model of melioidosis. 


\subsection{Carbohydrate composition of CPS III}

Glycosyl composition analysis was performed on the purified capsule by combined gas chromatography/mass spectrometry (GC/MS). GC/MS results indicated that CPS III is composed of galactose, glucose, mannose, xylose, and rhamnose residues, with the highest proportion of carbohydrate being galactose and glucose. Glycosyl linkage analysis was also performed [55]. The predominant glycosyl residue detected was a terminally-linked heptopyranosyl (t-Hep) at a percentage of 23.2. Other residues detected were a terminallylinked and a 4-linked glucopyranosyl (t-Glcp) (4-Glcp) at percentages of 14.6 and 10.8, respectively [55].

\section{Conclusion}

Although significant advances have been made in the field, melioidosis continues to be a public health concern in many regions of the world [59]. Completion of the sequencing of the $B$. pseudomallei genome has revealed potential virulence determinants and comparative genomics between the genomes of B. pseudomallei, B. thailandensis, and B. mallei species has contributed to a better understanding of the organism. Further studies are ongoing to define the pathogenesis of $B$. pseudomallei and to identify effective vaccine candidates and diagnostic targets $[54,59]$.

To obtain virulence determinants unique to $B$. pseudomallei, we used subtractive hybridization between this organism and a related nonpathogenic organism, $B$. thailandensis. Analysis of the subtractive hybridization library revealed that B. pseudomallei contains a number of DNA sequences that are not found in B. thailandensis. One of the subtraction clones, pDD1015, demonstrated weak homology to a glycosyltransferase, WbpX, from $P$. aeruginosa [43]. The insert from pDD1015 was cloned into a mobilizable suicide vector for insertional inactivation of the glycosyltransferase gene in wild-type B. pseudomallei. The resulting strain, SR1015, was markedly less virulent than the parent strain in an animal model. We determined that SR1015 harbored a mutation in a glycosyltransferase gene involved in the production of a capsular polysaccharide which we subsequently designated as CPS I. We then identified the operon involved in the biosynthesis and transport of this capsular polysaccharide (CPS I) [42]. The genes identified encode for proteins that are similar to proteins involved in the biosynthesis and export of capsular polysaccharides, particularly those involved in the production of group 3 capsular polysaccharides. Group 3 capsules include the E. coli $\mathrm{K} 10$ capsule and may also include the $H$. influenzae group b capsule and the capsule produced by N. meningitidis serogroup B [8]. Group 3 capsules are always coexpressed with $\mathrm{O}$ serogroups, are not thermoregulated, are transported by an $\mathrm{ABC}-2$ exporter system, and do not contain the $k p s U$ and $k p s F$ genes, and usually the gene clusters map near the serA locus [8]. Thus far, no serA locus that is associated with the type I O-PS cluster was identified, but this polysaccharide is coexpressed with $\mathrm{O}$ antigen and lacks the $k p s U$ and kpsF genes, and genes encoding for a putative ABC-2 transporter have been identified. The genes involved in the production of group 3 capsules are organized into regions and are divergently transcribed. Regions 1 and 3 are generally conserved and contain 
genes involved in export of the polysaccharide. These regions flank region 2, which contains the biosynthetic genes and is not conserved between serotypes [4]. The genetic organization of the CPS I is also similar to that of other capsule gene clusters in that the genes are organized into more than one transcriptional unit and appear to be divergently transcribed [42].

The polysaccharide with the structure -3)-2-O-acetyl-6-deoxy- $\beta$-D-manno-heptopyranose-(1was originally isolated and characterized as an O-PS component of LPS in B. pseudomallei and was designated type I O-PS [35]. However, our results suggested that this polysaccharide is a capsule rather than an O-PS moiety. The genes involved in the production of this capsule demonstrated strong homology to the genes involved in the production of capsular polysaccharides in many organisms, including N. meningitidis, $H$. influenzae, and E. coli. In addition, the export genes associated with this cluster are not associated with the previously characterized O-PS gene cluster [36]. Western blot analysis of proteinase $\mathrm{K}$ cell extracts and silver staining showed that this polysaccharide has a high molecular mass $(200 \mathrm{kDa})$ and lacks the banding pattern seen with O-PS moieties. This conclusion was further supported by another group of researchers that demonstrated this polysaccharide is a capsule rather than an O-PS component of LPS because it lacks a lipid A moiety and was not capable of macrophage activation [49]. Studies by our laboratory have indicated that mutants in the production of the core oligosaccharide of the LPS are still capable of producing this polysaccharide [48]. Based on the above criteria and the genetic similarity to group 3 capsules, we proposed that this polysaccharide is a capsule.

Virulence genes of a number of pathogenic bacteria are located on pathogenicity islands (PAIs), regions on the bacterial chromosome that are present in the genome of pathogenic strains but rarely present in those of nonpathogenic strains. The PAIs may range in size from about $30 \mathrm{~kb}$ to $200 \mathrm{~kb}$ and often differ in $\mathrm{G}+\mathrm{C}$ content from the remaining bacterial genome; the PAIs are often associated with the carriage of many virulence genes. These genetic units are often flanked by direct repeats and may be associated with tRNA genes or insertion sequence (IS) elements at their boundaries. They may also be associated with the presence of mobility genes, such as IS elements, integrases, transposases, and origins of plasmid replication. These DNA regions are considered to be unstable in that they may be subject to deletion with high frequency or undergo duplications and amplifications [7]. A number of PAIs have been described for both gram-positive and gram-negative bacteria, and the application of subtraction hybridization has been used to successfully identify such genetic elements [7]. The subtractive hybridization that was carried out between $B$. pseudomallei and $B$. thailandensis led to the identification of a number of sequences that were found to be A-T rich compared to the rest of the B. pseudomallei chromosome. This, combined with the fact that insertional mutagenesis of the glycosyltransferase gene identified by this method resulted in an avirulent strain, suggests that we may have identified DNA sequences from a putative PAI and that the capsular polysaccharide gene cluster may be located on this island. It is possible that B. pseudomallei, B. mallei, and B. stabilis acquired DNA encoding for capsule as well as other potential, yet unidentified virulence factors by horizontal transfer recently in evolution. B. pseudomallei and B. mallei are known to contain IS elements that are present in B. cepacia but not in B. thailandensis $[56,60]$. 
Capsule production has been correlated with virulence in many bacteria, particularly those causing serious invasive infections of humans [61]. Our studies demonstrated that CPS I is critical for the virulence of $B$. pseudomallei $[42,50]$. A number of functions have been suggested for polysaccharide capsules: prevention of desiccation for transmission and survival, adherence for colonization, resistance to complement-mediated phagocytosis and complement-mediated killing, and resistance to specific host immunity due to a poor antibody response to the capsule [4].

To establish a correlation between capsule production and clinical infection a number of $B$. pseudomallei strains isolated from clinical specimens were tested for CPS I production. All 55 strains tested were found to produce CPS I by western blot analysis [51]. In addition 10 strains of $B$. thailandensis were tested and found negative for CPS I production, confirming the importance of CPS I in virulence as well as clinical infection.

CPS I production by B. pseudomallei was shown to contribute to the persistence of the organism in the blood of the host. All CPS I mutants tested in the animal model could not be isolated from the blood following infection. The addition of purified capsule was shown to increase the virulence of the CPS I mutant strains SR1015 and SZ210 in the animal model. Differences in tissue distribution between wild type B. pseudomallei and SR1015 in infected hamsters indicated that SR1015 was cleared from the blood because the numbers of SR1015 in the blood of infected hamsters was 10,000-fold lower than that of wild type $1026 \mathrm{~b}$ and lower than the initial inoculum of $100 \mathrm{cfu} / \mathrm{ml}$ [50].

CPS I production was shown to be responsible for persistence in the blood by evasion of the complement cascade and the mechanism for this was determined to be through the reduction of $\mathrm{C} 3 \mathrm{~b}$ deposition and opsonophagocytosis. The addition of purified CPS I to serum bactericidal assays showed that the capsule contributes to increased resistance of serum sensitive strains lacking the O-polysaccharide moiety (O-PS) of LPS to the bactericidal effects of normal human serum. However, CPS I mutants themselves were not found to be serum sensitive because they still produced O-PS, which was previously shown to be responsible for serum resistance, because it prevents lysis by the MAC complex [36]. This led us to postulate that CPS I was affecting the complement cascade through some other mechanism and it was found that this mechanism was through the reduction of $\mathrm{C} 3 \mathrm{~b}$ deposition and opsonization [50]. Both Western blot analysis and immunofluorescence microscopy experiments using a mouse monoclonal antibody to human $\mathrm{C} 3 \mathrm{~b}$ demonstrated the inhibition of $\mathrm{C} 3 \mathrm{~b}$ deposition by CPS I. In both experiments C3b deposition was more pronounced on the surface of the CPS I mutant compared to wild type. Also evident was that some $\mathrm{C} 3 \mathrm{~b}$ deposition occurred in the wild type, but this was expected since bacterial capsules are known to allow the diffusion of some $\mathrm{C} 3 \mathrm{~b}$ to the bacterial surface and $B$. pseudomallei is capable of activating the alternative pathway of complement culminating in the formation of the MAC complex [36, 62]. The accumulation of C3b affects the amplification step of the complement cascade and therefore, the less C $3 \mathrm{~b}$ deposited the less C5a is generated for phagocyte recruitment [63]. This explains the increased clearance of CPS I mutants from the blood. This conclusion was supported by the fact that $B$. thailandensis, the non-pathogenic organism which lacks CPS I, has been shown to be serum 
resistant, but is not capable of establishing a bacteremia in the Syrian hamster model of acute melioidosis [36, 37, 42]. Effective opsonization of invading bacteria results in enhanced phagocytosis and clearance of organisms form the blood of an infected host [52]. Quantitative radiolabelled phagocytic assays were also performed to establish a correlation between opsonization of the bacteria and phagocytosis by polymorphonuclear leukocytes. In the presence of serum, the CPS I mutant was more readily phagocytosed than wild type [50].

The expression of CPS I in the presence of normal human serum was found to be significantly elevated, also confirming that this capsule contributes to survival in the host. The presence of CPS I enables B. pseudomallei to survive in the blood through the inhibition of complement factor $\mathrm{C} 3 \mathrm{~b}$ deposition and phagocytosis [50]. The presence of this capsule facilitates survival as well as spreading to other organs, which can explain the overwhelming septicemia that is common in culture-positive melioidosis patients [64]. Therefore CPS I production is critical to the virulence of B. pseudomallei and further research will enhance the development of preventative strategies for melioidosis since this polysaccharide is one of the components of a B. pseudomallei subunit vaccine [28, 65].

Sequence analysis of the genome of B. pseudomallei revealed the presence of four operons possibly involved in polysaccharide capsule biosynthesis. One of these operons, (CPS I), corresponded to the previously identified and characterized mannoheptose capsule that was shown to be responsible for virulence and comprises one of the currently proposed melioidosis and glanders subunit conjugate vaccine [28, 66, 42, 50,67]. The CPS I capsule cluster is present in the genome of $B$. mallei as well, but the complete cluster is not found in the genome of $B$. thailandensis $[56,57,68]$. This correlates with previous studies that have shown that this capsule is produced by $B$. mallei, but not by $B$. thailandensis $[36,37,42,56]$.

Three other putative capsule operons were identified by sequence analysis and all of these operons were found to be present in B. pseudomallei and B. thailandensis, but not B. mallei. Since these capsules are found in B. thailandensis and B. pseudomallei, they may be required for either survival in the host or in the environment; however, further studies are required to determine the roles of CPS II and CPS IV.

CPS III, located on chromosome 2, was found to contain 11 genes involved in the biosynthesis of a polysaccharide and was shown to be present in the genomes of $B$. pseudomallei and B. thailandensis, but not B. mallei. A mutation in the CPS III cluster did not affect production of CPS I and so it can be concluded that this operon encodes for gene products responsible for the biosynthesis of a separate capsule. CPS III was not found to contribute to the pathogenesis of B. pseudomallei. This capsule was not shown to be highly expressed in vivo by microarray analysis and was not required for virulence in the animal model. The CPS III mutant, SZ1829, which contains a mutation in the BPSS1829 gene as a result of insertional inactivation, was found to be as virulent in the animal model as wild type B. pseudomallei. The expression of this capsule was shown to be elevated when incubated in water, but suppressed in the presence of normal human serum [55]. The presence of the CPS III cluster in B. pseudomallei and B. thailandensis, both of which can 
survive for long periods in the environment compared to B. mallei, the increased expression of this capsule in water, and the low level of expression of this capsule in vivo, suggests that this capsule may contribute to the survival of B. pseudomallei in the environment [69].

Previous studies have demonstrated that B. pseudomallei produces three other capsular polysaccharides in addition to CPS I and these have been structurally characterized. One is an acidic polysaccharide with the structure, -3$)-2-O$-acetyl- $\beta$-D-Gal $p-(1-4)-\alpha$-D-Gal $p-(1-3)-\beta-$ D-Gal $p-(1-5)-\beta-\mathrm{D}-\mathrm{KDO} p-(2-$, which is recognized by patient sera [32]. The other two are: a branched 1,4-linked glucan polymer ((CP-1a) and a triple-branched heptasaccharide repeating unit composed of rhamnose, mannose, galatose, glucose, and glucoronic acid (CP2) [49]. Combined GC/MS analysis of CPS III revealed that the composition of this capsule demonstrates some similarity to the composition of the previously described capsule CP-2 composed of rhamnose, mannose, galactose, glucose, and glucoronic acid; however, the proportions of carbohydrate residues were not similar, and the CPS III capsule was also found to contain xylose and not glucoronic acid. In addition, CPS III was determined to be composed primarily of heptose [55]. Therefore it is evident that the capsule identified in this study is not one of the previously described capsule structures. Some of the previously characterized capsules produced by B. pseudomallei have been shown to be produced under unique conditions $[32,49]$. Strain variation, differences in expression of the capsules, and discrepancies between purification strategies may also explain why a number of capsules have been shown to be produced by this organism. Nevertheless, the genes BPSS 1825-1835 appear to be involved in the biosynthesis of a capsule with this composition. Further analysis by 2D NMR would be required to definitively establish a connection between CPS III and one of the other published structures.

Studies by another laboratory have also focused on the presence of these capsule clusters in B. pseudomallei. Sarkar-Tyson et al. identified two polysaccharide clusters, one of which corresponds to the CPS III presented in this paper, but the authors identified this cluster as type IV O-PS (2007). The type IV O-PS was found to be involved in virulence in a mouse model [70]. However, a mutant in this polysaccharide did not demonstrate any difference in hydrophobicity compared to wild-type, indicating that this polysaccharide does not contribute to making the cell surface more hydrophobic, which is an advantageous characteristic for some pathogenic bacteria. The differences in virulence compared to the current work can be attributed to the use of different animal models; however, all other data seem to indicate that this capsule is not required for virulence.

A study was recently published which outlines the identification of another capsule produced by B. pseudomallei [71]. This capsule was determined to be composed of 1,3-linked $\alpha$-D-mannose residues. This capsular polysaccharide was also found to be produced by $B$. mallei. The genes involved in the synthesis of this polysaccharide have not yet been identified and work is also underway to determine the role for this novel capsule in the pathogenesis of melioidosis and glanders.

B. pseudomallei is an environmental saprophyte often found in soil and stagnant water and incidence of the disease is high in rice farmers in Southeast Asia [22, 69]. This organism 
harbors a large genome which explains its ability to survive for long periods of time in the environment as well exist as a significant pathogen in both humans and animals. The presence of multiple polysaccharide clusters in the genome and the production of multiple capsule structures under differing conditions may contribute to the ability of this organism to adapt to a variety of conditions. As demonstrated in this study, capsule expression is dependent on the particular environment, which indicates that B. pseudomallei produces these capsules to promote a survival advantage either in the host or in the environment. Further studies aimed at characterizing the capsules of B. pseudomallei will be beneficial to understand the pathogenesis of this organism and to advance further vaccine development.

\section{Author details}

Shauna L. Reckseidler-Zenteno

Athabasca University, Athabasca, Alberta, Canada

\section{Acknowledgement}

The author would like to thank Dr. Donald Woods and all colleagues in the Burkholderia pseudomallei research community for their support and discussions over the years. The work described in this chapter was funded by the following sources: Department of Defense contract DAMD 17-98-C-8003, the Medical Research Council of Canada, the Canadian Bacterial Diseases Network of Centers of Excellence, Canadian Institutes for Health Research MOP 36343, and a Research Incentive Grant from Athabasca University. Carbohydrate analysis was conducted by the Complex Carbohydrate Research Center at the University of Georgia and this was supported in part by the Department of Energy-funded (DE-FG09-93ER-20097) Center for Plant and Microbial Complex Carbohydrates. Microarray analysis was performed at the Southern Alberta Microarray Facility.

\section{References}

[1] Whitfield C (1988) Bacterial Extracellular Polysaccharides. Can. J. Microbiol. 34: 415-420.

[2] Costerton J W, Irvin R T, Cheng K-J (1981) The Bacterial Glycocalyx in Nature and Disease. Annu. Rev. Microbiol. 35: 299-324.

[3] Whitfield C, and Valvano M (1993) Biosynthesis and Expression of Cell-surface Polysaccharides in Gram-negative Bacteria. Adv. Microbiol. Phys. 35: 135-146.

[4] Roberts I S (1996) The Biochemistry and Genetics of Capsular Polysaccharide Production in Bacteria. Ann. Rev. Microbiol. 50: 285-315.

[5] Robbins J B, McCracken G H, Gotschlich E C, Ørskov I, Hanson L A (1974). Escherichia coli K1 Capsular Polysaccharide Associated with Neonatal Meningitis. N. Engl. J. Med. 90: 267-271.

[6] Grados O, Ewing V M (1970) Antigenic Relationships between Escherichia coli and Neisseria meningitidis group B. J. Infect. Dis. 122: 100-103. 
[7] Hacker J, Blum-Oehler G, Mühldorfer I, Tschäpe H (1997) Pathogenicity Islands of Virulent Bacteria: Structure, Function and Impact on Microbial Evolution. Mol. Microbiol. 23: 1089-1097.

[8] Whitfield C, Roberts I S (1999) Structure, Assembly, and Regulation of Expression of Capsules in Escherichia coli. Mol. Microbiol. 31: 1307-1319.

[9] Frosch M, Müller D, Bousset K, Müller A (1992) Conserved Outer Membrane Protein of Neisseria meningitidis Involved in Capsule Expression. Infect. Immun. 60: 798-803.

[10] Roberson E, Firestone M (1992) Relationship between Dessication and Exopolysaccharide Production in Soil Pseudomonas sp. Appl. Environ. Microbiol.58: 1284-1291.

[11] Ophir T, Gutnick D (1994) A Role for Exopolysaccharide in the Protection of Microorganisms from Dessication. Appl. Environ. Microbiol. 60: 740-745.

[12] Berry A, DeVault J, Chakrabarty A (1989) High Osmolarity is a Signal for Enhanced algD Transcription in Mucoid and Nonmucoid Pseudomonas aeruginosa strains. J. Bacteriol. 171: 2312-2317.

[13] Costerton J W, Cheng K-J, Geesy G G, Ladd T I, Nickel J C et al. (1987) Bacterial Biofilms in Nature and Disease. Annu. Rev. Microbiol. 41: 435-464.

[14] Jenkinson F F (1994) Adherence and Accumulation of Oral Streptococci. Trends. Microbiol. 2: 209-212.

[15] Howard C J, Glynn A A (1971) The Virulence for Mice of Strains of Escherichia coli Related to the Effects of $\mathrm{K}$ antigens on their Resistance to Phagocytosis and Killing by Complement. Immunology 20: 767-777.

[16] Kim K, Kang, J, Cross A (1986) The Role of Capsular Antigens in Serum Resistance and In Vivo Virulence of Escherichia coli. FEMS Microbiol. Lett. 35: 275-278.

[17] Moxon E, Kroll J (1990) The Role of Bacterial Polysaccharide Capsules as Virulence Factors. Curr. Top. Microbiol. Immunol. 150: 65-85.

[18] Bhattacharjee A, Jennings H, Kenny C, Martin A, Smith I (1975) Structural Determination of the Sialic Acid Polysaccharide Antigens of Neisseria meningitidis Serogroups B and C with Carbon 13 Nuclear Magnetic Resonance. J. Biol. Chem. 250: 1926-1932.

[19] Vann W F, Schmidt M A, Jann B, Jann K (1981) The Structure of the Capsular Polysaccharide (K5 antigen) of Urinary Tract Infective Escherichia coli O10:K5:H4. A Polymer Similar to Desulfo-heparin. Eur. J. Biochem. 116: 359-364.

[20] Wyle F, Artenstein M, Brandt B L, Tramont E C, Kapser D L et al. (1972) Immunologic Response of Man to Group B Meningococcal Polysaccharide Vaccines. J. Infect. Dis. 126: 514-521.

[21] Dance, D A B (1991) Melioidosis: the Tip of the Iceberg? Clin. Microbiol. Rev. 4: 52-60.

[22] Leelarasamee A, Bovornkitti S (1989). Melioidosis: review and update. Rev. Infect. Dis. 11: 413-425.

[23] Chaowagul, W, White N J, Dance D A, Wattanagoon Y, Naigowit P, Davis T M, Looareesuwan S, Pitakwatchara N (1989). Melioidosis: a Major Cause of Community Acquired Septicemia in northeastern Thailand. J. Infect. Dis. 159: 890-899.

[24] Howe C, Sampath A, Spotnitz M (1971) The Pseudomallei Group: a Review. J. Infect. Dis. 124: 598-606. 
[25] Ip, M, Osterberg L G, Chau P Y, and Raffin T A (1995) Pulmonary Melioidosis. Chest 108: 1420-1424.

[26] Currie B J, Fisher D A, Anstey N M, Jacups S P (2000) Melioidosis: Acute and Chronic Disease, Relapse and Re-activation. Trans. R. Soc. Trop. Med. Hyg. 94: 301-304.

[27] White N J, Dance D A B, Chaowagul, W, Wattanagoon Y, Wuthiekanun V, Pitakwatchara N (1989) Halving of Mortality of Severe Melioidosis by Ceftazadime. Lancet 2: 697-701.

[28] Brett P J, Woods D E (1996) Structural and Immunologic Characterization of Burkholderia pseudomallei O-polysaccharide-flagellin Protein Conjugates. Infect. Immun. 64: 2824-2828.

[29] Bryan L E, Wong S, Woods D E, Dance D A, Chaowagul W (1994). Passive protection of diabetic rats with antisera specific for the polysaccharide portion of the lipopolysaccharide isolated from Pseudomonas pseudomallei. Can. J. Infect. Dis. 5: 170-178.

[30] Steinmetz I, Rohde M, Brenneke B (1995) Purification and Characterization of an Exopolysaccharide of Burkholderia (Pseudomonas) pseudomallei. Infect. Immun. 63: 3959-3965.

[31] Masoud, H, Ho M, Schollaardt T, Perry M B (1997) Characterization of the Capsular Polysaccharide of Burkholderia pseudomallei 304b. J. Bacteriol. 179: 5663-5669.

[32] Nimtz M, Wray V, Domke T, Brenneke B, Haussler S, Steinmetz I (1997). Structure of an Acidic Exopolysaccharide of Burkholderia pseudomallei. Eur. J. Biochem. 250: 608-616.

[33] Karawara K, Dejsirilert S, Ezaki T (1998) Characterization of Three Capsular Polysacharides Produced by Burkholderia pseudomallei. FEMS Microbiol Lett 169: 283-287.

[34] Knirel Y A, Paramonov N A, Shashkov A S, Kochetkov N K, Yarullin R G, Farber S M, Efremenko V I (1992) Structure of the Polysaccharide Chains of Pseudomonas pseudomallei Lipopolysaccharides. Carbohydr. Res. 233: 185-193.

[35] Perry M B, MacLean L L, Schollaardt T, Bryan L E, Ho M (1995) Structural Characterization of the Lipopolysaccharide $\mathrm{O}$ antigens of Burkholderia pseudomallei. Infect. Immun. 63: 3348-3352.

[36] DeShazer D, Brett P J, Woods D E (1998). The Type II O-antigenic Polysaccharide Moiety of Burkholderia pseudomallei Lipopolysaccharide is Required for Serum Resistance and Virulence. Mol. Microbiol. 30: 1081-1100.

[37] Brett P J, DeShazer D, Woods D E (1997) Characterization of Burkholderia pseudomallei and Burkholderia pseudomallei-like strains. Epidemiol. Infect. 118:137-148.

[38] Brett P J, DeShazer D, Woods D E (1998) Burkholderia thailandensis sp. nov., description of a Burkholderia pseudomallei-like species. Int. J. Syst. Bacteriol. 48: 317-320.

[39] Chaiyaroj S C, Kotrnon K, Koonpaew S, Anantagool N, White N J, Sirisinha S (1999) Differences in Genomic Macrorestriction Patterns of Arabinose-positive (Burkholderia thailandensis) and Arabinose-negative Burkholderia pseudomallei. Microbiol. Immunol. 43: 625-630.

[40] Wuthiekanun V, Smith M D, Dance D A, Walsh A L, Pitt T L, White N J (1996) Biochemical Characteristics of Clinical and Environmental Isolates of Burkholderia pseudomallei. J. Med. Microbiol. 45: 408-412.

[41] Trakulsomboon S, Dance D A B, Smith M D, White N J, Pitt T L (1997) Ribotype Differences between Clinical and Environmental Isolates of Burkholderia pseudomallei. J. Med. Microbiol. 46: 565-570. 
[42] Reckseidler S L, DeShazer D, Sokol P A, Woods D E (2001) Detection of Bacterial Virulence Genes by Subtractive Hybridization: Identification of Capsular Polysaccharide of Burkholderia pseudomallei as a Major Virulence Determinant. Infect. Immun. 69: 34-44.

[43] Rocchetta H L, Burrows L L, Pacan J C, Lam J S (1998) Three Rhamnosyltransferases Responsible for Assembly of the A-band D-rhamnan Polysaccharide in Pseudomonas aeruginosa: a Fourth Transferase, WbpL, is Required for the Initiation of both A band and B-band Lipopolysaccharide Synthesis. Mol. Microbiol. 28: 1103-1119.

[44] Mongkolsuk S, Rabibhadana S, Vattanaviboon P, Loprasert S (1994) Generalized and Mobilizable Positive-selective Cloning Vectors. Gene 143: 145-146.

[45] Steinmetz I, Reganzerowski A, Brenneke B, Haussler S, Simpson A, White N J (1999) Rapid Identification of Burkholderia pseudomallei by Latex Agglutination Based on an Exopolysaccharide-specific Monoclonal Antibody. J. Clin. Microbiol. 37: 225-228.

[46] Clarke B R, Pearce R, Roberts I S (1999) Genetic Organization of the Escherichia coli K10 Capsule Gene Cluster: Identification and Characterization of Two Conserved Regions in Group III Capsule Gene Clusters Encoding PolysaccharideTransport Functions. J. Bacteriol. 181: 2279-2285.

[47] Reeves P R, Hobbs M, Valvano M A, Skurnik M, Whitfield C, Coplin D, Kido N, Klena J, Maskell D, Raetz C R, Rick P D (1996) Bacterial Polysaccharide Synthesis and Gene Nomenclature. Trends Microbiol. 498: 495-503.

[48] Burtnick M N, Woods D E (1999) Isolation of Polymyxin B-susceptible Mutants of Burkholderia pseudomallei and Molecular Characterization of Genetic Loci Involved in Polymyxin B Resistance. Antimicrob. Agents Chemother. 43: 2648-2656.

[49] Isshiki Y, Matsuura M, Dejsirilert S, Ezaki T, Kawahara K (2001) Separation of 6-deoxyheptan from a Smooth-type Lipopolysaccharide Preparation of Burkholderia pseudomallei. FEMS Microbiol. Lett. 199: 21-25.

[50] Reckseidler-Zenteno S L, DeVinney R, Woods D E (2005). The Capsular Polysaccharide of Burkholderia pseudomallei Contributes to Survival in Serum by Reducing Complement Factor C3b Deposition. Infect. Immun. 73: 1106-1115.

[51] Reckseidler-Zenteno S L (2002) Role of the Burkholderia pseudomallei Capsule. University of Calgary. 228 p.

[52] Joiner K A, Brown E J, Frank M M (1984) Complement and Bacteria: Chemistry and Biology in Host Defense. Annu. Rev. Microbiol. 2: 461-491.

[53] Moore R A, Reckseidler-Zenteno S, Kim H, Nierman W, Yu Y, Tuanyok A, Warawa J, DeShazer D, Woods D E (2004) The Contribution of Gene Loss to the Pathogenic Evolution of Burkholderia pseudomallei and Burkholderia mallei. Infect. Immun. 72: 4172-4187.

[54] Holden M T, Titball R W, Peacock S J, Cerdeño-Tárraga A M, Atkins T, Crossman L C, Pitt T, Churcher C, Mungall K, et al. (2004) Genomic Plasticity of the Causative Agent of Melioidosis, Burkholderia pseudomallei. Proc. Natl. Acad. Sci. USA 101: 14240-14245.

[55] Reckseidler-Zenteno S L, Viteri D F, Moore R, Wong E, Tuanyok A, Woods D E (2010) Characterization of the Type III Capsular Polysaccharide Produced by Burkholderia pseudomallei. J. Med. Microbiol. 59(12): 1403-1414. 
[56] DeShazer D, Waag D M, Fritz D L, Woods D E (2001) Identification of a Burkholderia mallei Polysaccharide Gene Cluster by Subtractive Hybridization and Demonstration that the Encoded Capsule is an Essential Virulence Determinant. Microb. Pathog. 30: 253-269.

[57] Yu Y, Kim H S, Chua H H, Lin C H, Sim S H, Lin D, Derr A, Engels R, DeShazer D et al. (2006) Genomic Patterns of Pathogen Evolution Revealed by Comparison of Burkholderia pseudomallei, the Causative Agent of Melioidosis, to Avirulent Burkholderia thailandensis. BMC Microbiol. 6: 46.

[58] Tuanyok A, Tom M, Dunbar J, Woods D E (2006) Genome-wide Expression Analysis of Burkholderia pseudomallei Infection in a Hamster Model of Acute Melioidosis. Infect. Immun. 74: 5465-5476.

[59] Cheng A C, Currie B J (2005) Melioidosis: Epidemiology, Pathophysiology, and Management. Clin. Microbio.1 Rev. 18: 383-416.

[60] Mack K, Titball R W (1998) The Detection of Insertion Sequences within the Human Pathogen Burkholderia pseudomallei which have been Identified Previously in Burkholderia cepacia. FEMS Microbiol. Lett. 162: 69-74.

[61] Boulnois G J, Roberts I S (1990) Genetics of Capsular Polysaccharide Production in Bacteria. Curr. Top. Microbiol. Immunol. 150: 1-18.

[62] Egan, A M, Gordon, D L (1996) Burkholderia pseudomallei Activates Complement and is Ingested but not Killed by Polymorphonuclear Leukocytes. Infect. Immun. 64: 4952-4959.

[63] Densen P (1995) Complement. In: Mandell G L, Bennett J E, Dolin R, editors. Principles and Practice of Infectious Diseases. New York: Churchill Livingstone. pp. 58-78.

[64] Supputamongkol Y, Rajchanuwong A, Chaowagul W, Dance D A B, Smith M D, Wuthiekanun V, Walsh A L, Pukrittayakamee S, White N J (1994) Ceftazadime vs. Amoxicillin/Clavulanate in the Treatment of Severe Melioidosis. Clin. Infect. Dis. 19: 846-853.

[65] Patel N, Conejero L, De Reynal M, Easton A, Bancroft GJ, Titball RW (2011) Development of Vaccines against Burkholderia pseudomallei. Front. Microbiol. 2: 198.

[66] Brett P J, Woods D E (2000) Pathogenesis of and Immunity to Melioidosis. Acta. Trop. 74: 201-210.

[67] Warawa J, Woods D E (2002) Melioidosis Vaccines. Expert Rev. Vaccines 1: 477-482.

[68] Nierman W C, DeShazer D, Kim H S, Tettelin H, Nelson K E, Feldblyum T, Ulrich R L, Ronning C M, Brinkac L M, et al (2004) Structural Flexibility in the Burkholderia mallei Genome. Proc. Natl. Acad. Sci. USA 101: 14246-14251.

[69] Aldous P (2005) Tropical Medicine: Melioidosis? Never heard of it... Nature 434: 692-693.

[70] Sarkar-Tyson M, Thwaite J E, Harding S V, Smither S J, Oyston P C F, Atkins T P, Titball, R W (2007) Polysaccharides and Virulence of Burkholderia pseudomallei. J. Med. Microbiol. 56: 1005-1010.

[71] Heiss C, Burtnick M N, Wang Z, Azadi P, Brett P J (2012) Structural Analysis of Polysaccharides Expressed by Burkholderia mallei and Burkholderia pseudomallei. Carbohydrate Res. 349: 90-94. 